\title{
Early Specialization versus Sport Diversification in Youth Athlete-Article Review
}

\author{
Philip Dugas* \\ Adjunct Instructor, University of Western States, USA \\ *Corresponding author: Philip Dugas, Adjunct Instructor, University of Western States, USA

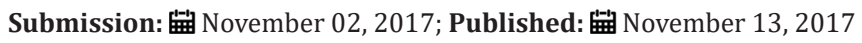

\section{Opinion}

Sport specialization is an increasingly dangerous epidemic that is having a negative effect on amateur athletes and the sports they play. The idea of participating in sports is to develop well-round and diverse youth by promoting self-esteem, leadership, and relationship building with teammates as well as developing athletic abilities to be successful in a particular sport. Sport specialization seems to have stemmed from an increase in participation and rise in competitive nature of youth sport. Competition in youth sport helps to build character and teaches how to deal with adverse situations. However, the push to win by adults has led to an increased number of young athletes playing a particular sport year round in a competitive environment. Sport specialization is defined as specific year round training in a specific sport with the exclusion of all other sport activities [1]. The reason behind this seems to be the allure of college scholarships and large professional contracts. The reality is only a small percentage of high school athletes are able to compete at the collegiate level and an even small number continue professionally.

There are several key issues highlighted in the article with regards to the increase in sport specialization. Motivation and interest in a particular sport can lead to a desire to only participate in that sport as the athlete's ability level and success increase. This can also lead to burnout, which is defined as physical and emotional exhaustion from the demands of an athlete's sport [1]. An increased risk of injury can easily occur when one activity is continuously complete without proper rest. Young athletes are still growing. This development is not seen in professional athletes as the body is more mature and can handle the stress that is placed on muscles and joints. The article mentions several overuse injuries being seen in baseball such as "Little League Elbow" and "Little League Shoulder". Little League Baseball, with the help of Dr. James Andrews, a lead surgeon in elbow and shoulder injuries, has developed a pitch limit to try and curb these types of injuries. However, young athletes who are specializing in baseball, for instance, may be playing on multiple teams throughout the year. The body is unable to get the proper rest and recovery time leading to more injuries.

The article concludes that sport diversification and nonspecialization in a sport is important in helping to develop coordination in young athletes. Playing multiple sports allows an athlete to develop different skills that can aid in success in other areas. This also can lead to less burnout and allow youth sports to flourish and remain competitive and an outlet for activity for all young athletes.

\section{References}

1. Curso T (2017) Early specialization versus diversification in youth sport. Personal Training Quarterly. 